\title{
Towards Dynamic Natural Interaction Ensembles
}

\author{
Bashar Altakrouri \\ Ambient Computing Group \\ Institute of Telematics \\ University of Luebeck \\ Luebeck, Germany \\ altakrouri@itm.uni-luebeck.de
}

\author{
Andreas Schrader \\ Ambient Computing Group \\ Institute of Telematics \\ University of Luebeck \\ Luebeck, Germany \\ andreas.schrader@itm.uni-luebeck.de
}

\begin{abstract}
Exploring the potential of whole body in motion is inevitably important for natural interactions. People are expected to use different body parts to simultaneously interact with multiple interactions techniques. Therefore, interactive ecosystems in interactive spaces become a real challenge. We have identified three closely related issues to be solved for better adoption of natural interactions in ambient systems: assessment of anthropometric physical abilities and disabilities, interaction ensembles and orchestration, and finally community-based designing and sharing of interactions. In this paper, we present an integrated concept for realizing interaction in ambient systems using natural interaction ensembles. Interaction modalities from different devices are tailored at runtime to maximize the adoption of interactive systems according to the users' physical abilities, needs, and context.
\end{abstract}

Ambient Assisted Living, Natural Interactions, Kinetic Interactions, Anthropometry.

\section{INTRODUCTION}

Pervasive computing technologies have paved the ground for the development of future interactive environments consisting of a plethora of interconnected smart objects realizing new contextaware services in a seamlessly integrated physical and virtual world. This imposes new challenges for human computer interaction $(\mathrm{HCl})$. The goal of $\mathrm{HCl}$ research for pervasive environments is to create user-friendly interaction means for essentially invisible technology. Technology should therefore adapt to the natural interaction abilities and practices of humans.

Human users will continue to interact with pervasive systems using physical body interactions and intermediaries, because major body activities such as touching, holding, and moving physical objects are the foundation of the long evolution of tool use in the human species. Similarly, voice based communication will continue to be used due to its essential part of our culture. Therefore, interaction using Natural Interfaces (NI) is frequently being proposed solution to support a flow of (inter-)action patterns in the hybrid world similar to the human patterns in the physical space.

The definition of natural interaction varies in the literature as noted by lacolina et al. (2010). Nevertheless, those definitions generally refer to the use of users' natural abilities, practices, and activities to control interactive systems. Such definitions inherently include activities such as but not limited to gestures, physical and virtual objects manipulations, body movements, and postures (lacolina et al. (2010)). NI resembles closely forms of human's communicative abilities (Abowd and Mynatt (2000)) and enables more natural and intuitive communication between people and all kinds of sensor-based devices, to enforce interactions that would "feel right". NI definition can be shortly devised from Wachs et al. (2011) as voice-based and kinetic-based interactions.

The improved integration of sensing and actuating technologies into commodity devices has set a strong ground for $\mathrm{NI}$ to be preponderant in pervasive environments such as experience centers and museums (Fogtmann et al. (2008)). The authors' research interest is currently focused on kinetic-based $\mathrm{NI}$, therefore other types of interactions are excluded intentionally from the discussion in this paper. As natural interactions between the physical and virtual spaces widely take place by means of gestures, manipulation and tangible artifacts as defined earlier, it is important to acknowledge that the core concepts covered in this paper can be still be applied to relevant and closely related interaction types in ambient spaces such as tangible interactions, interaction with 3D interfaces, ambient interactions, etc. 
The remainder of this paper includes an introduction to kinetic-based interactions, $\mathrm{NI}$ in pervasive environments, and $\mathrm{NI}$ and human physical abilities. Section 2 covers in detail the concept of anthropometric framework for $\mathrm{NI}$ ensembles. Section 3 presents the system design, issues and challenges. Sections 4 and 5 present some identified research challenges and conclusions respectively.

\subsection{Kinetic-based interactions}

Kinetic interactions are caused and characterized by motion and movement activities, e.g., running, walking, or dancing. They are natural and interesting for simulating physical activities and providing eyes-free interactions, i.e. interacting confidently in the absence of graphical feedback (Oakley and Park (2007)), for controlling devices. Hand-based kinetic interactions, for example, utilize tilting for scrolling photos, shaking for moving dices realistically, and hand gestures for drawing. Gesture-based interactions, another form of kinetic interactions that vary greatly in form and usability, promise new natural interaction techniques and lead to gesture-based systems as reported by (Rico and Brewster (2010)).

Different classifications of gesture interactions are proposed by literature. Herein we elect devicebased and body-based gesture classifications. The first refers to any gesture involving direct touching or moving of a typical interaction medium such as a mobile device. The later refers to any gesture involving direct movements of the body without the use of a typical interaction medium.

Researching this type of interaction is a challenging task. To our best knowledge, the literature still lacks published research on motion-based interaction primitives classification and design space of motion-based interactions, despite some recent research effort to end-user elicitation of motion gestures as reported by (Ruiz et al. (2011)). The authors provided a strong evidence for the importance and acceptability of kinetic-based NI, as a high percentage of respondents (up to $82 \%$ ) were willing to use motion-based gestures at least occasionally.

\subsection{NI in pervasive environments}

$\mathrm{NI}$ foster a set of important interaction qualities (Wachs et al. (2011)) including high accessibility, engagement, familiarity, easiness, intuitiveness (clear cognitive association with the functionality performed), come as you are, ubiquity and wearability without requiring long periods of learning and adaptation. Particularly, $\mathrm{NI}$ is able to solve a number of challenging aspects in pervasive systems:
- overcoming physical handicaps,

- exploring big data,

- and finally accessing and conveying information, meaning, and intentions while maintaining high sterility, where users are able to embrace such new, alternative interfaces and interactions.

Despite the novel research contribution in this area in the last few years, the fusion of $\mathrm{NI}$ techniques into ensembles of interaction techniques is still a rather unexplored area. The combination of hand and foot input for example has gained only little attention according to Daiber et al. (2011). Much of the literature focuses on using a limited part of the body. Interactive systems that incorporate the gross motor skills and utilize the kinesthetic sense have not been thoroughly investigated despite the growing number of implementation examples (Fogtmann et al. (2008)).

There is a strong emerging motivation to explore new potential in designing for the whole body in motion as in Kinesthetic Interactions by (Fogtmann et al. (2008)). Against this background, users are expected to interact with multiple interaction techniques simultaneously employing multiple body parts and different motor skills. Hence, $\mathrm{NI}$ is expected to not only play individually but also to play as part of an ensemble. We therefore endeavor to investigate the theoretical concept of $\mathrm{NI}$ ensembles and the potential realization technologies, which we believe will be part of the enabling technology for interactive pervasive systems.

Interaction with ambient systems is becoming increasingly more challenging as user population grows to include users with varying intrinsic sensorimotor capabilities, ranging from injuries, ageing, or other disorders. Interest in specially tailored applications for health related sensorimotor deficits have come to the fore.

Lately, home care research and industry are opting for more intuitive support for elderly and disabled people i.e. elderly people with physical limitations are actively using Wii for fun and rehabilitations (Bhuiyan and Picking (2009)). Nevertheless, this effort is still considered modest as vast research studies, i.e. surface computing, are still made for the general audiences with little focus on older adults (Piper et al. (2010)). Reviewing the literature reveals an extensive effort in the area of user interfaces adaptation in terms of context modeling, user modeling, automatic generation of interfaces, etc.

One of the well-established concepts is plasticity (Calvary et al. 2000). This concept refers to the capacity of an interactive system to tolerate changes in the context of use while retaining usability based on adapting the graphical user 
interface according to three factors (input, output, and platform). The WWHT framework (Rousseau et al. (2006)), on the other hand, is based on a rulebased system, which matches different communication channels to a given context model based on 4 levels of adaptation (What, Which, How, Then.).

Interface adaptation is a hot topic in $\mathrm{HCl}$ and covering more concepts is out of the scope of this paper. Nevertheless, most available adaptation approaches fail to satisfy four enduring of challenges drawn from the natural characteristics of ambient environments, namely heterogeneity, distributivity, dynamic media mobility, and user mobility (Pruvost et al. (2011)). Moreover, most adaptation approaches focus on interfaces issues such as information presentation but not the interaction per se.

There is currently a growing interest in investigating interaction adaptation. Recent work by Pruvost et al. (2011) focuses on interaction adaptations in ambient environments. They have suggested the concept of interaction ontology where semantic information about the interface, user and the context are used for interaction adaptation. They focus mainly on the structural adaptation of user interfaces and the adaptation of running interaction dialogs.

\subsection{NI and human physical abilities}

It is vital that anthropometric based analysis of $\mathrm{NI}$ leads to match users' physical abilities and disabilities to the current environment and interaction context. This match is very essential for designing interactions "for all" instead of focusing on a limited population percentile.

One of the most demanding user populations for $\mathrm{NI}$ is the senior citizen population, due to the notable effect of ageing in one's physical and motor abilities. The performance of interaction tasks is defined by the frequency of use, discretionary usage, computer familiarity, user knowledge, general abilities, physical abilities, and skills.

Elderly adults experience an overall slowing of movement and major problems with fine motor activity and coordination often resulting in inaccessible interfaces (e.g., mobile interfaces) according to Kane et al. (2009). NI are affected with wide range of physical impairments and disabilities including visual, hearing, and mobility impairments such as arthritis, paralysis, and Parkinson's disease contribute to vast range of symptoms affecting kinetic interactions greatly such as limited range of motion, pain, tremors, impaired balance, gait, etc.
Fogtmann et al. (2008) call for conceptual frameworks to identify unexplored possibilities when designing interactive systems addressing the body in motion. Hence, the theoretical gist of our research proposal is to study anthropometric driven ensembles of natural interaction techniques.

This proposal herein argues that the concept of $\mathrm{NI}$ ensembles is a necessary adaptive interaction enabler and a major technological player in interactive ambient assisted living systems. This paper presents our ideas, motivation, and the current work progress.

\section{ANTHROPOMETRIC FRAMEWORK FOR NI ENSEMBLES}

We propose STAGE framework for anthropometric driven ensembles of $\mathrm{NI}$ techniques. Interaction modalities from different devices are tailored at runtime to maximize the adoption of interactive systems according to the users' physical abilities, needs, and context.

STAGE utilizes detailed anthropometric data and human ability profiles for maximizing the usability of kinetic-based $\mathrm{NI}$ for acting on the stage of an ambient environment.

\subsection{The disability challenge and interactions}

A lot of devices specifically exist to support people with motor impairments such as oversized trackball mouse, and adaptive keyboard (for non-reliable muscle control and lack of precise movements, e.g., tremors). A study by Kane et al. (2009) on mobile interactions with motor-impaired people nevertheless reports a clear mismatch between the available devices and abilities of the motorimpaired participants, since none of them used accessibility mobile devices. One participant reported successful use of accessibility keyboards designed for children to interaction with her home PC. Nevertheless, she rejected the use of other accessibility devices such as mobile phones in public. Another participant illustrated some privacy issues with using a portable magnifier in public preventing her from interacting with a phone screen in public.

Obviously, designing $\mathrm{NI}$ devices is challenging because it does not fully explore the potential of motor interaction, even when optimized for considered impairments. This is mainly due to the following restrictions. First, these devices are usually designed with a specific impairment in mind but still compromising the variation of degrees of disabilities. Second, these devices are usually context-agnostic; resulting in one-design-fits-all approach e.g., the average car seat height fits almost nobody. 


\subsection{Natural interaction ensembles and orchestration}

Wachs et al. (2011) presented very useful interaction qualities that can be used to measure interactions against each other when applied in different contexts. This is to avoid the extremely poor use of the potential of the human's sensory and motor systems as in human operated machinery (e.g., automobile) as noted in Fogtmann et al. (2008).

We suggest de-coupling the close binding between devices, interaction methods, and applications. Alternatively, we suggest to utilize dynamic compositions of $\mathrm{NI}$ ensembles, assembled and configured based on user capabilities and situational context in an ad-hoc manner. Hence, the STAGE vision fosters soft-wired applications and devices. By using adaptive $\mathrm{NI}$ ensembles, the limitations of the static binding can be overcome, and one of the most challenging requirements in pervasive environments, the "come as you are", can be addressed. Moreover, mismatch problems between user's needs and device's offers can be avoided by employing the best matching $\mathrm{NI}$ to the given context, hence user independence (acceptability by permitting customizability) and usability as required by Wachs et al. (2011) are inherently enhanced.

STAGE in ambient environments is a runtime environment for natural interaction techniques and ensembles deployment and delivery based on a number of anthropometric and physical ability matching algorithms. STAGE treats each $\mathrm{NI}$ as a standalone interaction interpreter entity called Interaction Plugin (IP). We define Interaction Plugin as "an executable component in ambient interactive systems that encapsulates a single natural interaction technique with a set of interaction tasks as input and delivers higher level interaction primitives to applications based on specific interaction semantics". Therefore, IPs allow for $\mathrm{NI}$ techniques to be discoverable, exportable, exchangeable, plug-able, and sharable. We refer to interaction tasks as the unit of an entry of information by the user and occur repeatedly such as position, select, etc. Moreover, we refer to interaction primitives as the basic interaction units that glue between physical $1 / O$ devices and interaction and consumed by application such as panning, pinching, swipe, tap, etc.

Interaction Ensemble on the other hand is defined as "multiple interaction plugins grouped together to adapt the available interaction resources and possibilities to the user's physical context and abilities". So far, interaction ensembles are identified as being useful in 5 different cases (Figure 1): (i) full-similar substitution (replace an IP with another IP with the same set of interaction primitives and interaction tasks). This is useful when a better implementation or specially tailored IP to a particular disability or situation is available.

(ii) full-different substitution (replace an IP with another IP with the same set of interaction primitives but different interaction tasks). This case can be illustrated when two interaction tasks exist but with different nature (e.g., one based on rotation and the other based on linear movement.) Due to situational disability for example one of them is more accessible by the user or less affected by user's disabilities.

(iii) full-similar re-composition (replace an IP with composite set of interaction primitives with the same interaction tasks from multiple IPs).

(iv) full-different re-composition (replace an IP with composite set of interaction primitives with different interaction tasks from multiple IPs)

(v) partial re-composition (substitute partial set of interaction tasks from other IPs). This can be illustrated when for example only limited sets of interaction tasks are utilized by other IPs. For example, the user is capable of performing all interaction tasks other than the selection task by hand rotation. The system suggests other similar interaction tasks possible without hand rotation.

Ensembles will enforce better performance and integration of users within their known physical abilities and will also be increasingly useful in physical therapy and rehabilitation, e.g., maintaining and improving mobility, flexibility, strength, gait speed, and quality of life.

\subsection{Community-based designing and sharing of NI}

Community-based designing and sharing of $\mathrm{NI}$ are very important in order to easy the use of $\mathrm{NI}$ in application development, enhancing application adaptability, and promoting wide deployment of $\mathrm{NI}$ based applications. To our knowledge, there is no research specifically targeted at community-based creation and sharing for natural interaction techniques (as Interaction Plugins). This puts STAGE forward to be the first framework to study this concept rigorously. STAGE aims at communitybased description of $\mathrm{NI}$ techniques and contexts, supporting both ambient interactive system designers and application developers.

The presentation and readability of interactions are of equal importance to users, designers, and interaction recognition systems. Currently, most 
research papers use written descriptions, graphical sketches, and videos for the transmission and preservation of $\mathrm{NI}$ techniques. Videos, for example, are very powerful to show relationships between movements and excel in transmitting emotion. Even though fields that relay heavily on movement description, such as drama, and dance, do find the aforementioned methods useful, they avoid relaying on them heavily due to a number of inherent problems associated with those methods such as:
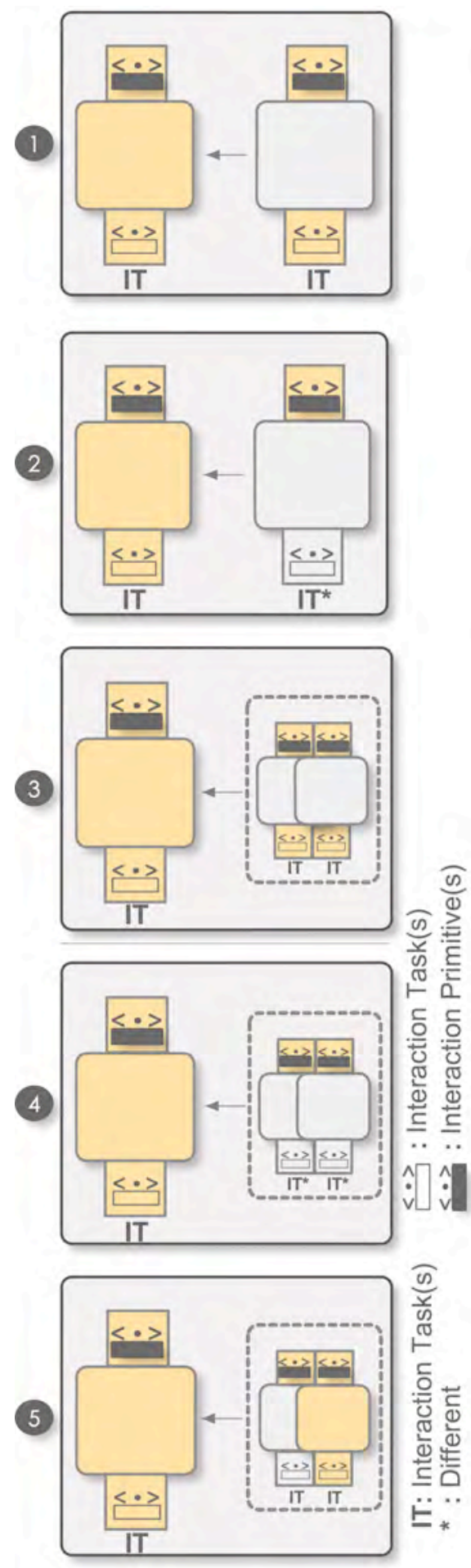

Figure 1: 5 Useful cases for NI ensembles
- inability to capture detailed description of the movements required,

- affected greatly by the production quality (e.g., videos are affected by lighting conditions and filming angle),

- inability to illustrate timing perfectly (e.g., parallel movements may be obscured by each other),

- and inability to utilize different medium to convey the movement (e.g., movements presented in a sketch are only provided in that form).

For successful transmission (sharing) and preservation (description) of $\mathrm{NI}$ techniques, recording and analyzing physical movement methods should be applied. Our research fosters the use of Labanotation (Kinetography) as a system for documenting physical movements required by $\mathrm{NI}$. Labanotation is a system of analyzing and recording movement, originally devised by Rudolf Laban in the 1920's. It is then further developed by Hutchinson and others at the Dance Notation Bureau (Hutchinson (2005)). Labanotation is used in fields traditionally associated with the physical body, such as dance choreography, physical therapy and drama.

Labanotation comprises a symbolic notation where symbols for body movements are written on a vertical "body" staff. Even though this system is very relevant to $\mathrm{HCl}$ research, only few research projects have demonstrated the use of this system to describe interaction techniques such as (Loke et al. (2005)). This can be the result of many reasons including but not limited to the researchers' lack of familiarity with reading and writing labanotation, the lack of tools for editing labanotation for interaction design, and limited recognition of the importance of documenting and sharing $\mathrm{NI}$ techniques. In STAGE, we do not only use Labanotation, but we exceed and extend the adoption of Labanotation in $\mathrm{NI}$ interaction design with anthropometric and physical ability profiles which is very important for adapting to the user's physical context in action.

Labanotation as a recording system for $\mathrm{NI}$ movements is very useful and has a number of relevant features such as it:

- is an extensive and flexible notation system,

- is easy to read and write (once familiarity is gained with the notation),

- is very logical and systematic,

- $\quad$ specifies movements from very simple and high level description to very specific movement description,

- has a great expressive power due to it's comprehensive symbol set, 
- enables choice for designers, about what they represent as significant and relevant aspects of movement.

The richness of the current Labanotation model serves wide range of purposes but at the same time requires enormous learning effort and results into an arbitrary complex notation to read. While preserving the extensibility and richness of the notation, we have opted for a subset of the notation to reduce its complexity and simplify its readability. To this end, the Labanotation subset models interaction sequences to include different parallel and sequential movements of body parts governed by the Labanotation score, which insure accurate time and sequencing of actions.

Labanotation as a graphical language is very powerful for human readers. It is nevertheless not readable by machines as to our knowledge there is no published research or standards on machinereadable representations for Labanotation adapted by the community. MovementXML was presented in a master thesis (Hatol (2006)) but it was neither dedicated to natural interaction techniques nor was the project completed. Therefore, part of our current work is to create XML (Extensible Markup Language) representation for Labanotation in order to be able to adapt the system in our proposed approach. We are planning to then extend Labanotation descriptions with the physical ability profiles introduced in section 3.2.1. This combination will be one of the essential driving wheels in the NI matching and decision algorithm in the process of creating $\mathrm{NI}$ ensembles based on the physical context and physical abilities required by interactions.

The reminder of this section will illustrate briefly how Labanotation is used in our approach to model one interaction technique as an example. We have selected the DoubleFlip interaction technique, introduced by Ruiz and $\mathrm{Li}$ (2011), as a simple technique for illustration. The authors define this technique as "a unique motion gesture designed as an input delimiter for mobile motion-based interaction." The authors document the technique using the following written description "the user holds the phone right-handed, he rotates the phone along its long side so that the phone screen is away and then back". Moreover, they supported the description with an additional sketch as shown in Figure 2.
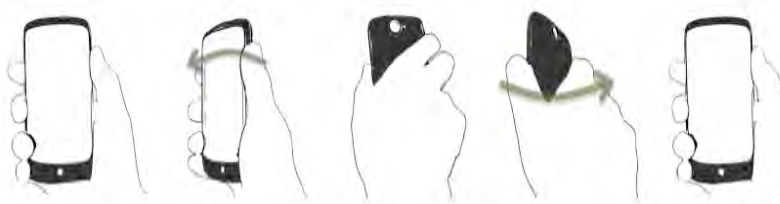

Figure 2: DoubleFlip interaction technique as in (Ruiz and $L i(2011))$
While it was relatively sufficient to relay on text and sketch descriptions for documenting this interaction, it is still relatively hard to explain clearly and insure that the user understands the steps to execute this technique. For example, neither the description nor the sketch clearly illustrates the manner and timing required for this interaction to work. Does the interaction work with very slow hand movement? Is there any break "pause" between the clockwise and counterclockwise movements? Etc.

We have modeled the same technique using Labanotation as in Figure 3. The figure is read as follows: (1) the body balance is equal on both legs and (2) stays that way through out the interaction. (3) The starting position of the right is at rest position along side the body and (4) the position of the lower arm to middle front, where arm and lower arm form "L" shape. Both positions are (5) held through out the interaction. Symbols (6) and (7) illustrate the starting position of the hand palm facing up. The wrist performs strong 180-degree counterclockwise rotation (8) and then returns back with palm facing up by a strong 180-degree clockwise rotation (9). Finally (10) the movement is split in terms of timing the described rotation movements.

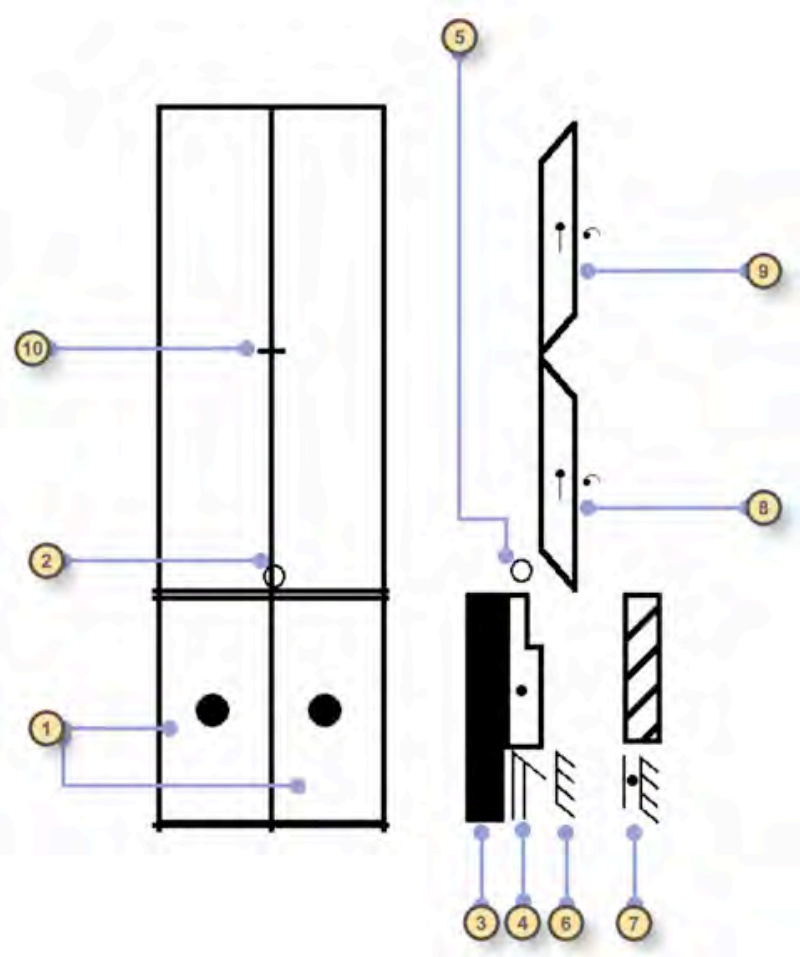

Figure 3: Labanotation representation for DoubleFlip interaction technique

In STAGE, an XML model of this technique is generated and extended with ability profile information needed to execute the model correctly by users. More detailed take on this part of our research is out of the scope of this paper but it is clear that $\mathrm{NI}$ technique transmission and 


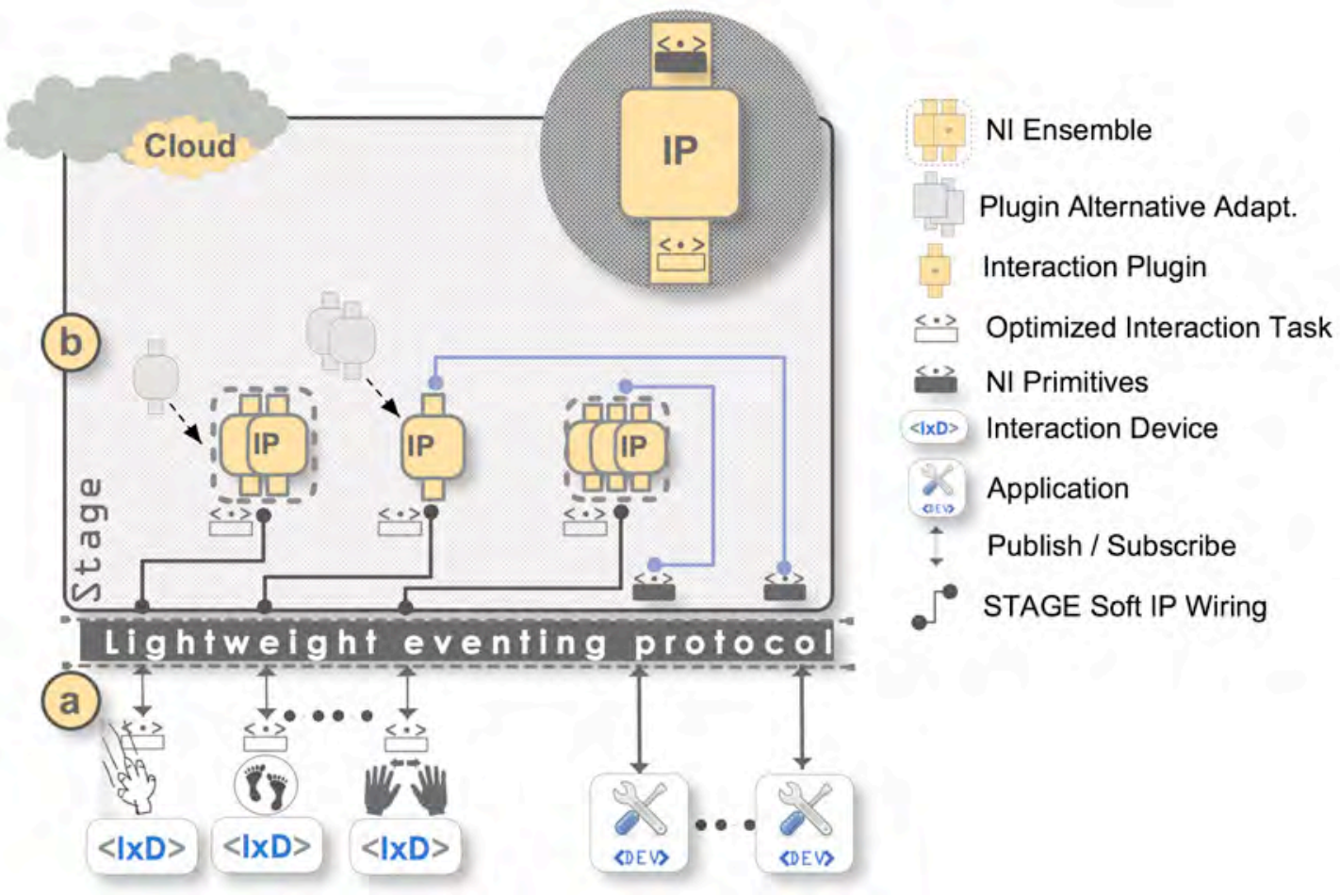

Figure 4: Stage conceptual diagram

preservation become more robust and standardized. More importantly, in the context of STAGE interactions steps and movement become well contained in a movement description entity, which can be also parsed using the STAGE ensemble engine (the core component of the STAGE runtime system, which is responsible to orchestrate and initiate $\mathrm{NI}$ ensembles).

\section{SYSTEM DESIGN, ISSUES, AND CHALLENGES}

\subsection{Conceptual View}

Edwards et al. (2010) explain that technological infrastructures that don't consider full range of human centered concerns present a fundamental tension for $\mathrm{HCl}$ and user experience designers. STAGE overcomes this problem by targeting developers and $\mathrm{HCl}$ designers equally. It avoids reductionist infrastructure design by taking a deep approach (Edwards et al. (2010)) to involve interaction and technically orientated metrics. Pervasive environments inevitably inherent crossplatform challenges. Thus STAGE adopts a cloudbased approach for hosting and processing $\mathrm{NI}$ ensembles. This imposes a number of technological challenges to investigate: managing on-device resources (low-level $\mathrm{NI}$ capture and preprocessing), eventing and networking problems, and addressing extensibility and modularity needs.

In Figure 4(a), both interaction devices (interaction providers) and applications (interaction consumers) are based on arbitrary technical platforms, built by interaction designers and by application developers respectively. Both are connected to STAGE via lightweight publish/subscribe eventing protocols such as Extensible Messaging and Presence Protocol (XMPP) aiming at a high level of interoperability and compatibility. STAGE prevails communication problems by avoiding bandwidth intense payloads e.g., images. It uses highly optimized interaction tasks data types based on an extended list of primitives including position, movement, rotation, etc., and optimized interaction primitive data types for the consuming applications such as selection, panning, etc. As indicated in Figure 4(b), interaction designers and $\mathrm{HCl}$ researchers create their IPs and publish them using STAGE interaction publishing front end. IPs are controlled by the Ensemble Controlling Unit. Moreover, interactions are provided by a single atomic IP or by an ensemble of IPs orchestrated by STAGE. Interactions provided by an IP may well have a number of implementation alternatives if multiple interaction resource provides alternative implementations of the same IP as shown in section 2.2.

\subsection{Assessment of physical abilities and disabilities}

Major part of our current effort on STAGE is channeled to develop an interaction-in-context matching algorithm to activate and ensemble the best matching $\mathrm{NI}$ for a given user's context. The 


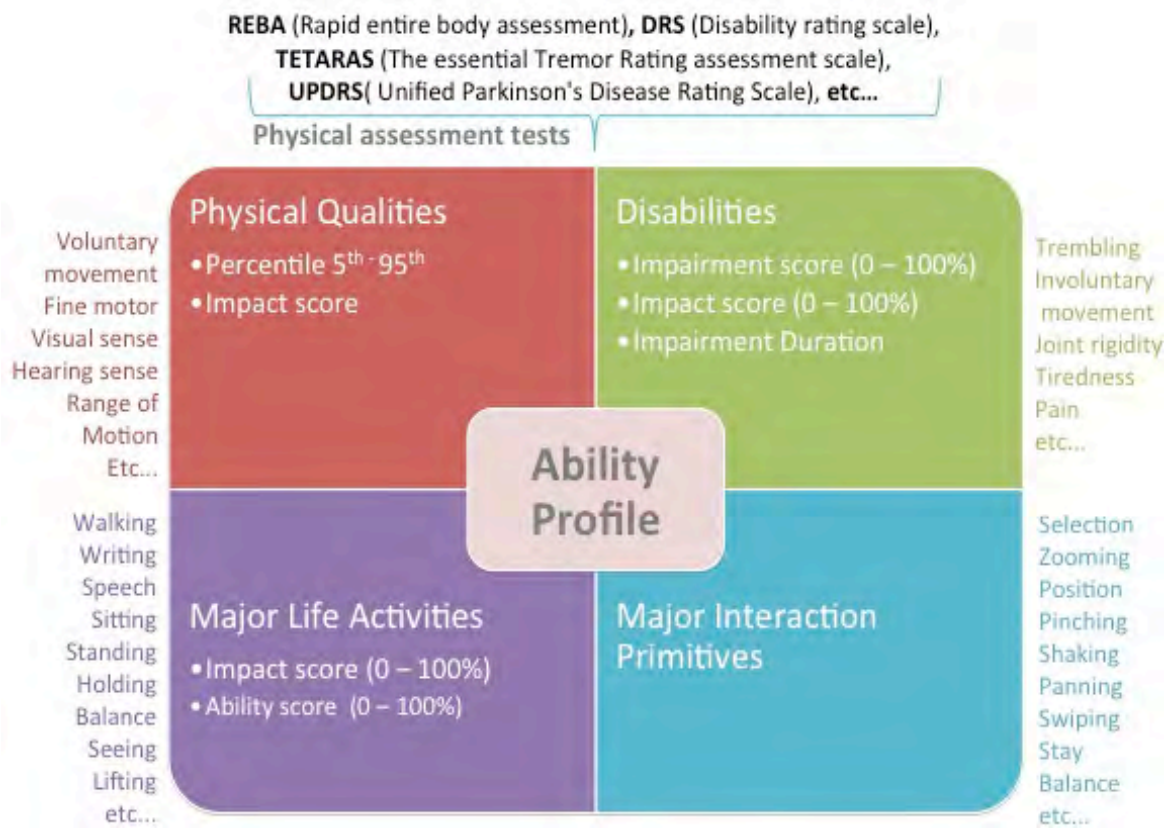

Figure 5: Ability Profile

algorithm utilizes three main concepts: ability profiling, interaction profiling, and ability matrix.

\subsubsection{Ability profile and interaction profile}

Ability profile (Figure 5) contains quantified anthropometric abilities tested by specialists or the user herself. It is defined by four key elements:

Physical qualities: indicate the required physical skills for the interactions e.g., voluntary movement and range of motion.

Disabilities (quantified by impact scores): indicate the quality and duration of the interaction. Impairment symptoms are normally quantitatively rated with physical assessment and rating scales. Documenting physical disabilities research provides a strong background in this direction. The core matching algorithm in the ensemble engine utilizes then different physical assessment and rating scales to reason about the severity of the symptoms and their impact on the interaction quality.

Major life activities: In our model, each interaction is linked to one or more major life activities such as walking, balancing, seeing, lifting, etc. The ability to perform the required activity is a good indication on the ability to perform the respective interaction.

Major interaction primitives: linked to physical abilities such as selecting, zooming, positioning, shaking, panning, etc. On the other side, interaction profiles shortly describe the interaction generally by indicating the main body part or parts involved in the interaction, type of movement, range of movement, capture method, disabilities, major life activities, interaction primitives, and hardware.

\subsubsection{Ability matrix}

The ability matrix presents disabilities and their direct impact on the major life activities. Major life activities may be affected by one or more disabilities but with different degree. Therefore, the impairment score is used to prioritize the impact of each disability of the interaction quality. The ability matrix (Figure 6) should be developed by physical assessment and diagnoses specialists e.g., physical therapists and physicians, and will be used as a tool by interaction designers and $\mathrm{HCl}$ researchers while designing $\mathrm{NI}$.

\section{DISCUSSION OVER RESEARCH CHALLENGES}

Within this section we examine four challenges relevant to the presented concept of this paper.

- Interaction performance and viability: Performance measures (QoS) such as response time and latency of $\mathrm{NI}$ ensembles is affected by the design and implementation of STAGE. Therefore, special attention should be paid to meet an acceptable threshold to hold a smooth, useful, and meaningful user experience.

- Interaction and ability profile matching: There should be a semantic matching between the physical profiles of users and interactions in order to match the user's context and fulfill the interactions primitive required. Physical action required by the interaction should be made part of the interaction semantic. Moreover, the flexibility of movement description in 
Labanotation triggers challenging aspects such as the complexity of describing movement in fine details and the danger to lose important aspects of interaction in rough descriptions. It is important for the interaction designers to illustrate and stress the main and essential movements for the interactions.

- Interaction sharing: Authoring interactions independently from a specific application is a very challenging aspect in interaction design. Moreover, interaction should be natively designed for orchestration and fusion with others, therefore high adaptability should be maintained.

- Application development: Designing softwired applications is more challenging due to $\mathrm{NI}$ resource management issues such as $\mathrm{NI}$ priority management, conflict resolution, affordance, user involvement and preferences.

\begin{tabular}{|c|c|c|c|c|}
\hline 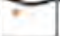 & $\because$ & 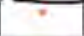 & Motor response & \multirow{11}{*}{ 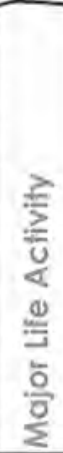 } \\
\hline & & - & Grasping & \\
\hline & - & & Araising & \\
\hline & $*$ & & Standing & \\
\hline & * & & Leying & \\
\hline & * & & Sitting & \\
\hline & $\cdot$ & & Jumping & \\
\hline - & * & - & Movement & \\
\hline \multirow{3}{*}{ 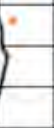 } & $*$ & $\cdot$ & Sensing & \\
\hline & $*$ & & Walking & \\
\hline & $*$ & & Running & \\
\hline $\mathrm{rms}$ & Legs & Hand & & \\
\hline$\cdot$ & $\cdot$ & $\cdot$ & Reduced ROM & \multirow{10}{*}{ 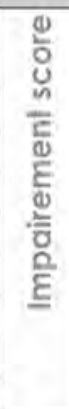 } \\
\hline$=$ & . & $\cdot$ & Pain/swallen & \\
\hline 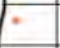 & $\cdot$ & $\cdot$ & Lack coordination & \\
\hline - & * & - & Weakness & \\
\hline+ & $*$ & + & Move Rigidity & \\
\hline \multirow[t]{2}{*}{$\cdot$} & $\cdot$ & $\cdot$ & Trembling & \\
\hline & & & Gait / leaning & \\
\hline \multirow[t]{2}{*}{ - } & $*$ & & Gait / freeze & \\
\hline & & & Toe Tapping & \\
\hline 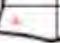 & $\therefore$ & 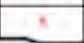 & Posture instability & \\
\hline
\end{tabular}

Figure 6: Ability Matrix (excerpt)

\section{CONCLUSION}

This work is part of the community effort towards utilizing our body in motion for better integrated interactions in ambient systems. We call for Natural Interaction Ensembles as an adaptive model for natural interactions based on physical abilities and anthropometric qualities. This approach opens many important questions regarding developing, deploying, adopting, and sharing interaction techniques. We believe that this area of research could provide new and powerful means of interactions in ambient spaces and can be similarly applied to closely related types of interactions such as tangible interactions.

\section{ACKNOWLEDGMENTS}

This work was partially supported by the Graduate School for Computing in Medicine and Life Sciences funded by Germany's Excellence Initiative [DFG GSC 235/1].

\section{REFERENCES}

Abowd, G. D. and Mynatt, E. D. (2000) Charting past, present, and future research in ubiquitous computing. ACM Trans. Comput.-Hum. Interact. 7, 29-58.

Bhuiyan, M. and Picking, R. (2009) Gesturecontrolled user interfaces, what have we done and what's next?. Technical report. Centre for Applied Internet Research (CAIR), wrexham, uk.

Calvary, G., Coutaz, J. and Thevenin. D. (2000) Embedding plasticity in the development process of interactive systems. First Workshop on Resource Sensitive Mobile $\mathrm{HCl}$, Conference on Handheld and Ubiquitous Computing, HU2K, bristol, 2000.

Daiber, F., Schöning, J. and Krüger, A. (2011) Towards a framework for whole body interaction with geospatial data. In D. England (ed), Whole body interaction, Human-Computer Interaction Series. Springer London, Iondon.

Edwards, W. K., Newman, M. W. and Poole, E. S. (2010) The infrastructure problem in $\mathrm{HCl}$. CHI'10: 28th International Conference on Human Factors in Computing Systems, atlanta, usa, 1015 April 2010, 423-432. ACM, new york.

Fogtmann, M. H., Fritsch, J. and Kortbek, K. J. (2008) Kinesthetic interaction: revealing the bodily potential in interaction design. OZCHI'08: 20th Australasian Conference on ComputerHuman Interaction: Designing for Habitus and Habitat, cairns, australia, 8-12 December 2008, 89-96. ACM, new york.

Hatol, J. (2006) Movementxml: a representation of semantics of human movement based on labanotation. Simon Fraser University. Master's thesis.

Hutchinson, A. (2005) Labanotation the system of analyzing and recording movement. Routledge, new york and london.

lacolina, S., Lai, A. and Scateni, R. (2010) Natural interaction and computer graphics applications. Eurographics Italian Chapter Conference, genova, italy, 141-146. Eurographics.

Kane, S. K., Jayant, C., Wobbrock, J. O. and Ladner, R. E. (2009) Freedom to roam: a study of mobile device adoption and accessibility for people with visual and motor disabilities. Assets 09: 11th international ACM SIGACCESS 
Conference on Computers and Accessibility, pittsburg, usa, 26-28 October 2009, 115-122. ACM, new york, USA.

Loke, L., Larssen, A. T. and Robertson, T. (2005) Labanotation for design of movement-based interaction. Second Australasian Conference on Interactive Entertainment, sydney, australia, 2325 November 2005, 113-120. Creativity \& Cognition Studios Press, sydney, australia,.

Oakley, I. and Park, J. (2007) Designing eyes-free interaction. HAID'07: 2nd International Conference on Haptic and Audio Interaction Design, seoul, korea, 121-132. Springer-Verlag berlin, heidelberg,.

Piper, A. M., Campbell, R. and Hollan, J. D. (2010) Exploring the accessibility and appeal of surface computing for older adult health care support. CHI'10: 28th International Conference on Human Factors in Computing Systems, atlanta, usa, 1015 April 2010, 907-916. ACM, new york.

Pruvost, G., Heinroth, T., Bellik, Y. and Minker, W. (2011) User interaction adaptation within ambient environments. In T. Heinroth and W. Minker (eds), Next generation intelligent environments: ambient adaptive systems. Springer Boston, usa.
Rico, J. and Brewster, S. (2010) Usable gestures for mobile interfaces: evaluating social acceptability. CHI'10: 28th International Conference on Human Factors in Computing Systems, atlanta, usa, 10-15 April 2010, 887896. ACM, new york.

Rousseau, C., Bellik, Y., Vernier, F. and Bazalgette, D. (2006) A framework for the intelligent multimodal presentation of information. Signal processing - special section: multimodal human-computer interfaces, volume 86 issue 12, 3696-3713.

Ruiz, J. and Li, Y. (2011) Doubleflip: a motion gesture delimiter for mobile interaction. CHI'11: 29th International Conference on Human Factors in Computing Systems, vancouver, british columbia, 7-12 May 2010, 2717-2720. ACM, new york.

Ruiz, J., Li, Y. and Lank, E. (2011) User-defined motion gestures for mobile interaction. $\mathrm{CHI} 11$ : 29th International Conference on Human Factors in Computing Systems, vancouver, british columbia, 7-12 May 2010, 197-206. ACM, new york.

Wachs, J. P., Kölsch, M., Stern, H. and Edan, Y. (2011) Vision-based hand-gesture applications. ACM communications, volume 54 issue 2, 6071. 